\title{
Palladium-Catalyzed Three-Component Diaryl Sulfone Synthesis Exploiting the Sulfur Dioxide Surrogate DABSO**
}

\author{
Edward J. Emmett, Barry R. Hayter, and Michael C. Willis*
}

Aryl, heteroaryl, and alkenyl sulfones play a prominent role in organic and medicinal chemistry. Not only are they versatile intermediates in organic synthesis ${ }^{[1]}$, but they are also of particular pharmaceutical relevance, and exhibit an extensive and broad range of biological activities (Scheme 1). ${ }^{[2]}$ In addition, sulfone-containing polymers dis-
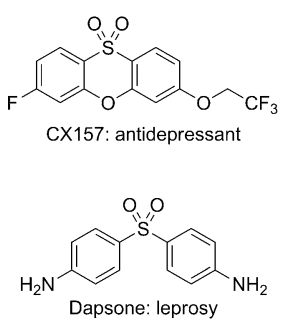

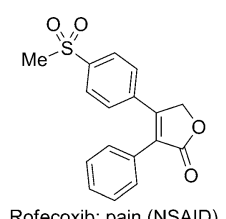

Rofecoxib: pain (NSAID)

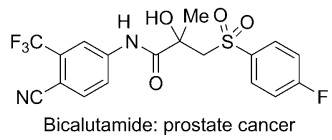

Scheme 1. Pharmaceuticals featuring an aryl sulfone unit.

play novel properties as materials. ${ }^{[3]}$ Although there are a variety of methods known for the preparation of sulfones, the majority of these processes feature associated limitations. For example, oxidation of the corresponding sulfide relies on functional-group compatibility with oxidizing agents and the availability of catalysts to promote selectivity for sulfone formation over sulfoxide formation; in addition, the preparation of the required sulfide substrate often involves the use of foul-smelling thiols. ${ }^{[4,5]}$ Friedel-Crafts-type sulfonylation of arenes is limited not only by the generally harsh reaction conditions, but also by the inherent regioselective bias

[*] E. J. Emmett, Prof. M. C. Willis

Department of Chemistry, University of Oxford

Chemistry Research Laboratory

Mansfield Road, Oxford, OX1 3TA (UK)

E-mail: michael.willis@chem.ox.ac.uk

Homepage: http://mcwillis.chem.ox.ac.uk/MCW/Home.html

Dr. B. R. Hayter

Oncology Innovative Medicines, AstraZeneca

Alderley Park, Macclesfield, Cheshire, SK10 4TG (UK)

[*** This work was supported by the EPSRC and AstraZeneca. DABSO is a solid, bench-stable complex formed between DABCO and two sulfur dioxide molecules.

0 Supporting information for this article is available on the WWWW under http://dx.doi.org/10.1002/anie.201305369.

of (c) 2013 The Authors. Published by Wiley-VCH Verlag GmbH \& Co. $\mathrm{KGaA}$. This is an open access article under the terms of the Creative Commons Attribution License, which permits use, distribution and reproduction in any medium, provided the original work is properly cited. imposed by the electronic and steric properties of the arene substrate. ${ }^{[5,6]}$

In response to these limitations, the use of transitionmetal-catalyzed cross-coupling has been exploited as a route to sulfones. ${ }^{[7]}$ The most utilized has been copper catalysis, in which sodium sulfinates couple with aryl, heteroaryl, or alkenyl halides, ${ }^{[8]}$ or boronic acids. ${ }^{[9]}$ However, the requirement for stoichiometric phase-transfer additives, high temperatures, and the use of undesirable solvents or ionic liquids, are significant drawbacks. An analogous palladium-catalyzed approach has also been developed using (pseudo)halides as coupling partners, although it too features the use of stoichiometric additives and high temperatures, and consequently suffers from limited scope ${ }^{[10,11]}$ In addition to these problems, the variety of sulfones prepared by these metalcatalyzed methods is poor; only a small number of sodium sulfinates (typically methyl, phenyl, and $p$-tolyl) have been used to demonstrate the scope. This reflects the very limited commercial availability of sodium sulfinates, perhaps as a result of impractical or limited synthetic routes. Organometallic addition to sulfur dioxide gas ${ }^{[12]}$ (followed by cation exchange) and reduction of the sulfonyl chloride ${ }^{[12,13]}$ are two such examples, but both have shortcomings such as the harsh conditions associated with sulfonyl chloride formation or the use of toxic gaseous sulfur dioxide. Taken together, these limitations present a clear mandate for the development of a more general, modular synthesis of sulfones, with the versatility to allow ready variation of both groups attached to the $\mathrm{SO}_{2}$ linker. ${ }^{[14]}$

We have recently developed DABSO (1), which is a solid, bench-stable complex formed between 1,4-diazabicyclo[2.2.2] octane (DABCO) and two sulfur dioxide molecules, ${ }^{[15]}$ as an easy-to-handle surrogate for sulfur dioxide gas (Scheme 2). We have demonstrated its use in known reactions

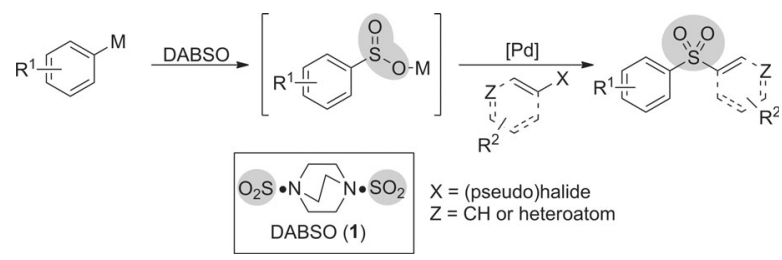

Scheme 2. A convergent three-component sulfone synthesis exploiting DABSO, an easy-to-handle solid replacement for sulfur dioxide gas.

of sulfur dioxide, ${ }^{[16]}$ as well as in a novel palladium-catalyzed aminosulfonylation of aryl, heteroaryl, and alkenyl halides to yield medicinally important sulfonamides. ${ }^{[17]}$ Herein, we report on a three-component convergent synthesis of 
a broad range of aryl, heteroaryl, and alkenyl sulfones, employing palladium catalysis and DABSO (Scheme 2).

Given our previous demonstration of the efficient addition of Grignard reagents to DABSO to form magnesium halide sulfinate salts, ${ }^{[16]}$ we envisioned that these would couple with (pseudo)halides to form sulfones in a similar fashion to the reported sodium salt examples using transition metal catalysis. Unfortunately, after testing a variety of catalytic conditions, we were not able to achieve this. Suspecting that this was due to the difference in the nature of the counter ions, we elected to study the addition of organolithiums to form the more closely related lithium sulfinates. We chose to explore palladium rather than copper catalysis because the reported conditions for sodium salts were the most amenable to our organolithium proposal. ${ }^{[10,11]}$ We were mindful, however, of the precedent for certain metal sulfinates to undergo palladium-catalyzed desulfonylative cross-couplings, whereby extrusion of $\mathrm{SO}_{2}$ from the palladium-bound sulfinate ultimately leads to a biaryl product. ${ }^{[18]}$

We began our investigations with the addition of $\mathrm{PhLi}$ solution to a suspension of DABSO in THF at $-40^{\circ} \mathrm{C}$. Pleasingly, HPLC analysis revealed that quantitative conversion to the lithium sulfinate $\mathbf{2}$ was achieved; only traces of benzene from deleterious protonation were detected. This crude sulfinate suspension was then combined with $\left[\mathrm{Pd}_{2}-\right.$ $\left.(\mathrm{dba})_{3}\right] \quad(\mathrm{dba}=$ dibenzylideneacetone $)$, XantPhos, $\mathrm{Cs}_{2} \mathrm{CO}_{3}$, $n \mathrm{Bu}_{4} \mathrm{NCl}$, and $p$-tolyl iodide; the conditions reported for sodium sulfinate coupling. ${ }^{[10]}$ Analysis of the crude ${ }^{1} \mathrm{H}$ NMR spectrum indicated the presence of the desired sulfone $\mathbf{3 a}$, albeit at a low conversion (see the Supporting Information). However, by switching to $\mathrm{Pd}(\mathrm{OAc})_{2}$, conversion increased to $65 \%$ and it was also found that the $n \mathrm{Bu}_{4} \mathrm{NCl}$ additive was effectively redundant. Postulating that the incomplete conversion was a result of the lower temperature used relative to that for the sodium salt, the solvent was switched from THF to 1,4-dioxane, and the reaction heated at $85^{\circ} \mathrm{C}$. Gratifyingly, a quantitative conversion was achieved (Scheme 3 ).

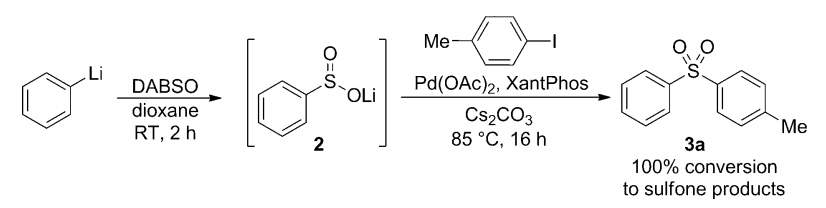

Scheme 3. Initial investigation of the generation and Pd-catalyzed coupling of lithium sulfinate 2 .

Unfortunately, upon purification, two different sulfone products were isolated. Desired sulfone 3a was present in $70 \%$ yield together with $23 \%$ of diphenylsulfone. From the observation that a phenyl group was always incorporated in place of the aryl halide fragment upon varying the organolithium component, we deduced that this side product was a result of aryl-phenyl exchange with the XantPhos ligand. Such exchange processes are known in arylation of electronpoor nucleophiles and in Suzuki couplings. ${ }^{[19-22]}$ Conditions for the palladium coupling step were fully explored, with the choice of base, additive, solvent, palladium source, and temperature being investigated. However, no improvement in selectivity whilst maintaining good conversion of starting material was observed. ${ }^{[22]}$ Finally, variation of the phosphine ligand was undertaken. We found that, in agreement with the work of Cacchi et al., the XantPhos backbone was key to reactivity ${ }^{[10]}$ many other ligands, including those with similar structures, such as DPEPhos, as well as alkyl phosphines (which cannot undergo this exchange process), demonstrated little or no activity. ${ }^{[22]}$ With the XantPhos architecture established as optimal, we next explored electronic and steric variations of this unique backbone. ${ }^{[23]}$ These ligands were evaluated under the cross-coupling conditions previously established (Table 1). Alkyl (entries 3-5) and sterically

Table 1: Evaluation of XantPhos-type ligands to improve selectivity through the suppression of aryl-aryl exchange. ${ }^{[a]}$

\begin{tabular}{|c|c|c|c|c|c|}
\hline \multirow[b]{2}{*}{ Entry } & \multirow{2}{*}{$\begin{array}{l}\text { Li } \\
\text { dioxane } \\
\text { RT, } 2 \text { h }\end{array}$} & \multirow[b]{2}{*}{ Pd source } & $\begin{array}{c}\begin{array}{l}\text { Pd source, } \mathrm{Cs}_{2} \mathrm{CO}_{3} \\
\text { ligand } 5 \mathrm{a}-\mathrm{f}\end{array} \\
\stackrel{\begin{array}{c}85^{\circ} \mathrm{C}, 16 \mathrm{~h} \\
\mathrm{X}=1 \text { or } \mathrm{Br}\end{array}}{\longrightarrow}\end{array}$ & $\underset{\mathrm{Br}}{\mathrm{Bh}}$ & 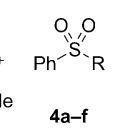 \\
\hline & & & $\mathrm{I} / \mathrm{Br}$ & Conversion $[\%]^{[d]}$ & $3 \mathrm{a}: 4 \mathrm{a}-\mathrm{f}^{[\mathrm{d}]}$ \\
\hline 1 & XantPhos & $\mathrm{Pd}(\mathrm{OAc})_{2}$ & I & 100 & $3: 1$ \\
\hline $2^{[b]}$ & XantPhos & {$\left[\mathrm{Pd}_{2}(\mathrm{dba})_{3}\right]$} & 1 & 100 & $2: 1$ \\
\hline 3 & $5 a$ & $\mathrm{Pd}(\mathrm{OAc})_{2}$ & 1 & - & - \\
\hline 4 & $5 b$ & $\mathrm{Pd}(\mathrm{OAc})_{2}$ & 1 & - & - \\
\hline $5^{[b]}$ & $5 b$ & {$\left[\mathrm{Pd}_{2}(\mathrm{dba})_{3}\right]$} & 1 & - & - \\
\hline 6 & $5 c$ & $\mathrm{Pd}(\mathrm{OAc})_{2}$ & 1 & 40 & $1: 1$ \\
\hline 7 & $5 d$ & $\mathrm{Pd}(\mathrm{OAc})_{2}$ & 1 & trace & - \\
\hline 8 & $5 e$ & $\mathrm{Pd}(\mathrm{OAc})_{2}$ & 1 & 95 & $3: 1$ \\
\hline 9 & $5 \mathrm{f}$ & $\mathrm{Pd}(\mathrm{OAc})_{2}$ & 1 & - & - \\
\hline $10^{[b]}$ & $5 \mathrm{f}$ & {$\left[\mathrm{Pd}_{2}(\mathrm{dba})_{3}\right]$} & 1 & 50 & $5: 1$ \\
\hline $11^{[c]}$ & XantPhos & $\mathrm{Pd}(\mathrm{OAc})_{2}$ & $\mathrm{Br}$ & 100 & $7: 2$ \\
\hline $12^{[b, c]}$ & $5 \mathrm{f}$ & {$\left[\mathrm{Pd}_{2}(\mathrm{dba})_{3}\right]$} & $\mathrm{Br}$ & 100 & $4: 1$ \\
\hline $13^{[c]}$ & $5 \mathrm{f}$ & $\mathrm{Pd}(\mathrm{OAc})_{2}$ & $\mathrm{Br}$ & 100 & $15: 1$ \\
\hline
\end{tabular}

[a] Reaction conditions: PhLi (1.4 equiv), DABSO (0.75 equiv), 1,4dioxane $(0.18 \mathrm{M})$ then $p$-tolyl halide (1 equiv, $0.35 \mathrm{mmol})$, palladium source $(10 \mathrm{~mol} \% \mathrm{Pd})$, ligand (10 mol\%), $\mathrm{Cs}_{2} \mathrm{CO}_{3}$ ( 1.5 equiv). [b] With $n \mathrm{Bu}_{4} \mathrm{NCl}$ additive (1.5 equiv). [c] Performed at $110^{\circ} \mathrm{C}$. [d] Determined by ${ }^{1} \mathrm{H}$ NMR spectrum integration to the nearest $5 \%$.

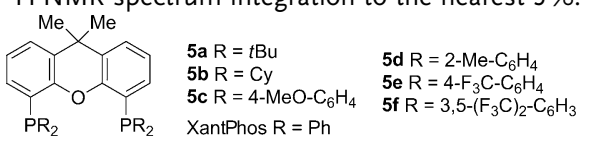

hindering aryl substituents (entries 3 and 7) on the XantPhos backbone led only to recovered starting material. As expected, a more electron-rich phosphine gave more arylphenyl transfer product (entry 6). ${ }^{[21]}$ Electron-poor phosphines, which have been reported to suppress transfer for the arylation of ureas, ${ }^{[20]}$ gave similar or a small increase in selectivity as hoped, but for the more selective reaction, the conversion was poor (entries 8-10). However, by switching to the aryl bromide substrate in place of the iodide substrate, and increasing the temperature of the reaction, full conversion was achieved with high selectivity when using the 3,5bis $\left(\mathrm{CF}_{3}\right)$-substituted XantPhos $5 \mathbf{f}$ (entry 13). Using these optimized conditions, the scope of the aryl bromide coupling partner was next investigated (Scheme 4).

$m$-Tolyllithium 6a was chosen to demonstrate the synthesis of sulfones that have not been prepared through 

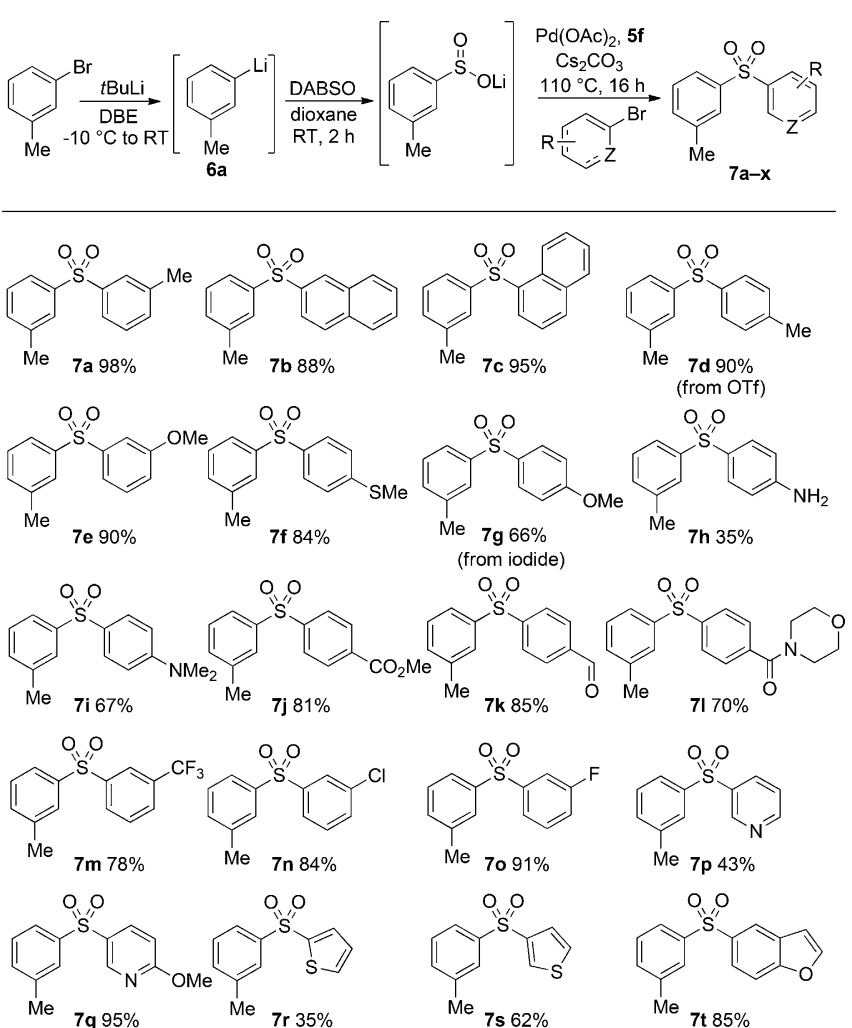

0,0

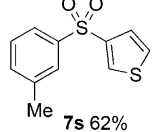

Me $7 p 43 \%$

O O

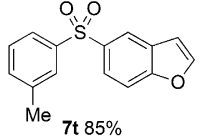

(s)
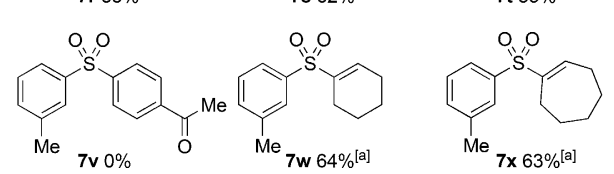

Scheme 4. Scope of the aryl halide component in the Pd-catalyzed preparation of sulfones through coupling with lithium sulfinates. Coupling conditions as described in Table 1, entry 13. [a] When using the alkenyl tosylate, $\mathrm{K}_{3} \mathrm{PO}_{4}$ ( 1.5 equiv) employed in the place of $\mathrm{Cs}_{2} \mathrm{CO}_{3}$

sodium sulfinate coupling. A stock solution was readily synthesized by using lithium-halogen exchange between butyllithium and 3-bromotoluene in dibutylether, which is a Lewis-basic solvent in which lithium-halogen exchange is kinetically facile and yet aryl lithiums are more stable to storage than in $\mathrm{Et}_{2} \mathrm{O}$ or $\mathrm{THF}^{[24]} \mathrm{A}$ wide range of aryl and heteroaryl bromides coupled in good to excellent yields (Scheme 4). Electron-rich substrates performed well, with C-, $\mathrm{O}-, \mathrm{S}-$, and N-based functionality all tolerated. Electron-poor aryl bromides also proved to be compatible, with ester, amide, aldehyde, chloro, and trifluoromethyl functional groups retained. The use of unprotected anilines, aryl fluoride, and napthyl derivatives demonstrated further scope. We established additional versatility by using heteroaromatic pyridyl, thiophenyl, and benzofuryl bromides, as well as using iodide or triflate substrates in place of bromide. Unfortunately, ketones and arenes bearing ortho substituents led to no reaction. Provided that $\mathrm{K}_{3} \mathrm{PO}_{4}$ was used in place of $\mathrm{Cs}_{2} \mathrm{CO}_{3}$, alkenyl tosylates could be successfully employed as the electrophilic coupling partner and allowed access to the corresponding aryl-alkenyl sulfone products. ${ }^{[11]}$

Next, we investigated the scope of the organolithium species (Scheme 5). Using 3-bromoanisole as the standard
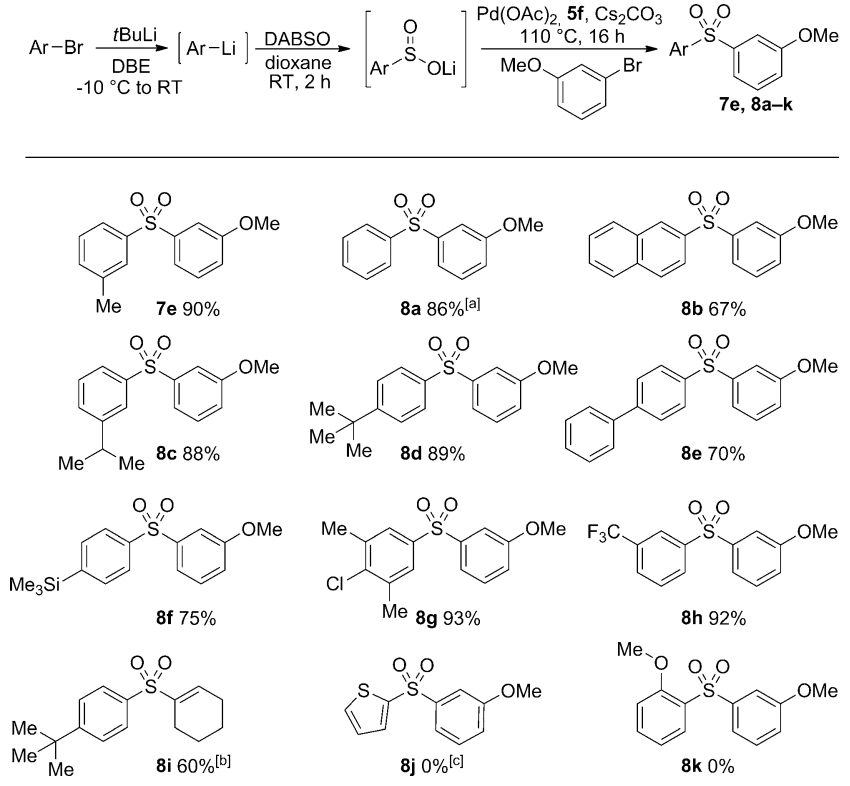

Scheme 5. Scope of the aryl lithium component in the Pd-catalyzed preparation of sulfones through the coupling of lithium sulfinates with aryl halides. Coupling conditions as described in Table 1, entry 13.

[a] Organolithium used as commercial solution. [b] From the corresponding alkenyl tosylate. [c] Biaryl formation observed.

coupling partner, a range of aryl lithiums were successfully subjected to the optimized conditions. Pleasingly, all the organolithiums prepared added, as expected, to DABSO to form the corresponding lithium sulfinate, and the subsequent palladium-catalyzed coupling worked well for a broad range of aryl sulfinates, including those with electron-donating, neutral, and electron-withdrawing functionality; trifluoromethyl, chloro, and silane functional groups were all successfully tolerated. Unfortunately, heterocyclic lithium 2-thienylsulfinate led to biaryl formation from desulfonylative coupling through $\mathrm{SO}_{2}$ extrusion, ${ }^{[18]}$ while ortho-substituted lithium 2-anisyl sulfinate resulted in the recovery of aryl bromide starting material.

A limitation of our presented methodology was the inability to include functionality positioned ortho to the sulfone moiety. To address this issue, we exploited the capacity of the sulfone functional group to direct ortho metalation. ${ }^{[25]}$ We demonstrated this through two regioselective ortho functionalizations of sulfone $\mathbf{8 d}$ by using the cooperative effect of meta-disposed directing groups to give 1,2,3-trisubsituted aryl sulfones $\mathbf{9}$ and $\mathbf{1 0}$ in good yields (not optimized, Scheme 6).

As a proof of the versatility and applicability of the presented methodology, we sought to demonstrate the synthesis of a significant target molecule. GSK-742457 (11) is a quinoline-based drug molecule that is currently under development for treating Alzheimer's disease. ${ }^{[26]}$ We envisaged disconnection to the known bis-halogenated quinoline derivative 12. ${ }^{[27]}$ In the forward direction, palladium-catalyzed sulfonylation of $\mathbf{1 2}$ would generate chloro derivative $\mathbf{1 3}$, and a subsequent palladium-catalyzed Buchwald-Hartwig amination would produce the target medicinal agent (Scheme 7). The proposed sequence was successfully achieved, with the 


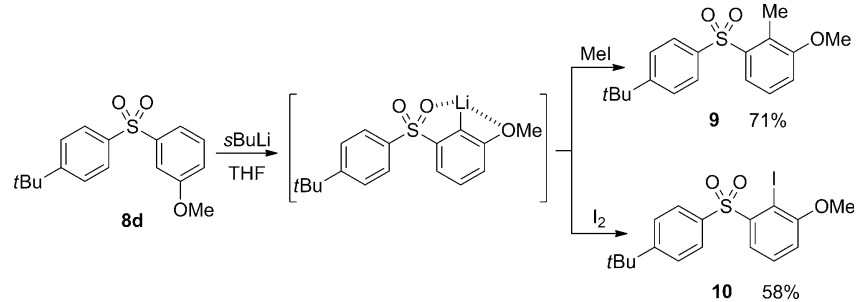

Scheme 6. Exploiting sulfonyl groups to direct ortho functionalization.

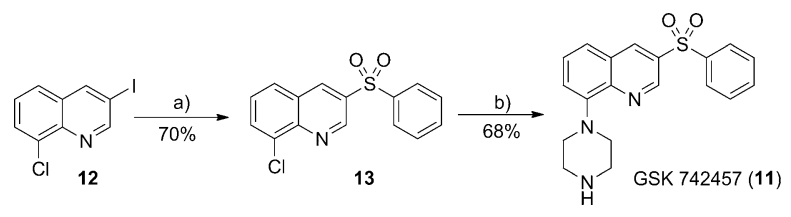

Scheme 7. Synthesis of GSK-742457 using the combination of a Pdcatalyzed aryl halide-lithium sulfinate coupling, and a Pd-catalyzed aryl halide amination. Conditions: a) Sulfonylation with aryl lithium (see Table 1, entry 13). b) $\mathrm{Pd}(\mathrm{OAc})_{2}$, RuPhos, $\mathrm{NaOtBu}$, piperazine, 1,4dioxane, $90^{\circ} \mathrm{C}, 16 \mathrm{~h}$. RuPhos $=2$-Dicyclohexylphosphino-2', $6^{\prime}$-diisopropoxybiphenyl.

sulfonylation being realized in $70 \%$ yield, and the amination in $68 \%$ yield (both not optimized). This is an attractive route to the target system via the bis-halogenated quinoline $\mathbf{1 2}$, which provides a suitable branch point for the rapid preparation of analogues.

In summary, we have demonstrated the synthesis of a broad range of aryl, heteroaryl, and alkenyl sulfones through a convergent three-component palladium-catalyzed coupling approach. This method allows the straightforward production of varied sulfones through the union of two readily available coupling partners; an aryl lithium species ${ }^{[28]}$ and aryl, heteroaryl, or alkenyl (pseudo)halides; with $\mathrm{SO}_{2}$ provided by the easy-to-handle bench-stable solid surrogate DABSO. The use of an electron-poor XantPhos-type ligand offers much improved yields over XantPhos itself. In addition, ortho functionality can be introduced by exploiting the ability of the sulfone group to direct ortho metalation. A demonstration of the utility of the methodology was achieved through the synthesis of a medicinal agent that is currently in development, utilizing a versatile bis-halogenated quinoline as the starting material. Finally, evidence for the nontrivial nature of the preparation of aryl-aryl (and related) sulfones is found in the observation that of the 36 sulfone products reported herein, more than $90 \%$ are novel compounds.

Received: June 21, 2013

Published online: October 2, 2013

Keywords: aryl lithium reagents - palladium - sulfones . sulfur dioxide $\cdot$ synthetic methods

[1] N. S. Simpkins, Sulfones in Organic Synthesis Pergamon Press, Oxford, 1993.
[2] a) P. Prasit, Z. Wang, C. Brideau, C. C. Chan, S. Charleson, W. Cromlish, D. Ethier, J. F. Evans, A. W. Ford-Hutchinson, J. Y. Gauthier et al., Bioorg. Med. Chem. Lett. 1999, 9, 1773; b) C. F. Sturino, G. O’Neill, N. Lachance, M. Boyd, C. Berthelette, M. Labelle, L. Li, B. Roy, J. Scheigetz, N. Tsou et al., J. Med. Chem. 2007, 50, 794; c) W. M. Wolf, J. Mol. Struct. 1999, 474, 113; M. Murár, G. Addová, A. Boháč, Beilstein J. Org. Chem. 2013, 9 , 173; d) N. Neamati, A. Mazumder, H. Zhao, S. Sunder, T. R. Burke, R. J. Schultz, Y. Pommier, Antimicrob. Agents Chemother. 1997, 41, 385; e) R. Ettari, E. Nizi, M. E. Di Francesco, M.A. Dude, G. Pradel, R. Vicik, T. Schirmeister, N. Micale, S. Grasso, M. Zappala, J. Med. Chem. 2008, 51, 988; f) E. C. A. Brot, D. K. J. Keefe, B. P. Haney, N. Metcalfe, G. J. Palmer, P. K. Isbester (CeNeRx BioPharma Inc.), US2008009542 (A1), 2008; g) Y. I. Zhu, M. J. Stiller, J. Am. Acad. Dermatol. 2001, 45, 420; h) P. F. Schellhammer, R. Sharifi, N. L. Block, M. S. Soloway, P. M. Venner, A. L. Patterson, M. F. Sarosdy, N. J. Vogelzang, J. J. Schellenger, G. J. Kolvenbag, Urology 1997, 50, 330.

[3] "Polysulfones": M. J. El-Hibri, S. A. Weinberg, Encyclopedia of Polymer Science and Technology, Wiley, New York, 2002.

[4] a) M. Jereb, Green Chem. 2012, 14, 3047; K. Bahrami, M. M. Khodaei, M. Sheikh Arabi, J. Org. Chem. 2010, 75, 6208; b) K. Schank, The Chemistry of Sulfones and Sulfoxides, Wiley, New York, 1988, Chapter 7.

[5] S. Oae, Organic Sulfur Chemistry, CRC, Boca Raton, FL, 1992.

[6] a) M. Ueda, K. Uchiyama, T. Kano, Synthesis 1984, 323; b) D. U. Singh, P. R. Singh, S. D. Samant, Tetrahedron Lett. 2004, 45, 9079; c) B. M. Graybill, J. Org. Chem. 1967, 32, 2931; d) S. J. Nara, J. R. Harjani, M. M. Salunkhe, J. Org. Chem. 2001, 66, 8616; e) C. G. Frost, J. P. Hartley, A. J. Whittle, Synlett 2001, 830; B. P. Bandgar, S. P. Kasture, Synth. Commun. 2001, 31, 1065; f) J. Marquié, A. Laporterie, J. Dubac, N. Roques, J.-R. Desmurs, J. Org. Chem. 2001, 66, 421.

[7] B. P. Bandgar, S. V. Bettigeri, J. Phopase, Org. Lett. 2004, 6, 2105.

[8] a) J. M. Baskin, Z. Y. Wang, Org. Lett. 2002, 4, 4423; b) W. Zhu, D. W. Ma, J. Org. Chem. 2005, 70, 2696; c) M. Bian, F. Xu, C. Ma, Synthesis 2007, 2951.

[9] a) M. L. Kantam, B. Neelima, B. Sreedhar, R. Chakravarti, Synlett 2008, 1455; b) F. Huang, R. A. Batey, Tetrahedron 2007, 63, 7667; c) A. Kar, I. A. Sayyed, W. F. Lo, H. M. Kaiser, M. Beller, M. K. Tse, Org. Lett. 2007, 9, 3405; d) H. Yang, Y. Li, M. Jiang, J. Wang, H. Fu, Chem. Eur. J. 2011, 17, 5652.

[10] S. Cacchi, G. Fabrizi, A. Goggiamani, L. M. Parisi, R. Bernini, J. Org. Chem. 2004, 69, 5608.

[11] D. C. Reeves, S. Rodriguez, H. Lee, N. Haddad, D. Krishnamurthy, C. H. Senanayake, Tetrahedron Lett. 2009, 50, 2870.

[12] "Sulfinic Acids and Derivatives": R. Schubart, Ullmann's Encyclopedia of Industrial Chemistry, Wiley-VCH, Weinheim, 2000.

[13] a) L. Kao Liu, Y. Chi, K.-Y. Jen, J. Org. Chem. 1980, 45, 406; b) N. Umierski, G. Manolikakes, Org. Lett. 2013, 15, 188.

[14] During the preparation of our manuscript, Manolikakes and Umierski published on the metal-free coupling of a broader range sodium sulfinates with diaryliodonium salts (Ref. [13b]). Whilst the progress towards metal-free couplings is attractive, the use of poorly atom-economical aryl iodonium salts and their associated problems with versatility towards using nontrivial aryl groups represents a significant constraint.

[15] P. S. Santos, M. T. S. Mello, J. Mol. Struct. 1988, 178, 121.

[16] H. Woolven, C. Gonzalez-Rodriguez, I. Marco, A. L. Thompson, M. C. Willis, Org. Lett. 2011, 13, 4876.

[17] a) E. J. Emmett, C. S. Richards-Taylor, B. Nguyen, A. GarciaRubia, B. R. Hayter, M. C. Willis, Org. Biomol. Chem. 2012, 10, 4007; b) B. Nguyen, E. J. Emmett, M. C. Willis, J. Am. Chem. Soc. 2010, 132, 16372.

[18] a) S. Sévigny, P. Forgione, Chem. Eur. J. 2013, 19, 2256; D. H. Ortgies, A. Barthelme, S. Aly, B. Desharnais, S. Rioux, P. 
Forgione, Synthesis 2013, 694; b) C. Zhou, Q. Liu, Y. Li, R Zhang, X. Fu, C. Duan, J. Org. Chem. 2012, 77, 10468.

[19] a) K. C. Kong, C. H. Cheng, J. Am. Chem. Soc. 1991, 113, 6313; b) F. E. Goodson, T. I. Wallow, B. M. Novak, Org. Synth. 1998 75,61 .

[20] A. G. Sergeev, G. A. Artamkina, I. P. Beletskaya, Tetrahedron Lett. 2003, 44, 4719 .

[21] B. C. Hamann, J. F. Hartwig, J. Am. Chem. Soc. 1998, 120, 3694.

[22] See the Supporting Information for proposed mechanism, and associated conditions screened.

[23] These were all prepared through double ortho lithiation of 9,9dimethylxanthene, followed by trapping with the appropriate chlorophosphine, according to reported procedures (see the Supporting Information).
[24] J. A. Schwindeman, D. E. Sutton, R. C. Morrison, S. S. Stryker (FMC Corporation), US 587813, 1996.

[25] a) M. Iwao, T. Iihama, K. K. Mahalanabis, H. Perrier, V. Snieckus, J. Org. Chem. 1989, 54, 24; b) J. Clayden, J. Senior, Synlett 2009, 2769.

[26] A. E. Gladwin (Glaxo Group Ltd.), WO2005040124 (A1), 2005.

[27] This bis-halogenated quinoline was synthesized from commercially available 8-chloroquinoline through an NIS iodination (see Ref. [26].

[28] For the recent use of aryl lithium species in efficient palladiumcatalyzed cross-couplings with aryl halides, see M. Giannerini, M. Fañanás-Mastral, B. L. Feringa, Nat. Chem. 2013, 5, 667. 